\title{
Nonrecurrent Laryngeal Nerve: A Case Report and Literature Review
}

\author{
Enrico Maria Amadei M.D.", Christopher Fabbri M.D. and Marco Trebbi M.D. \\ Department of Otorhinolaryngology and Audiology, Infermi Hospital, Rimini, Italy.
}

\section{ABSTRACT}

We report the case of a patient submitted to a right hemithyroidectomy for a follicular adenoma, when we found a nonrecurrent laryngeal nerve. This is a really rare anatomical presentation that can seriously compromise the integrity and preservation of the inferior laryngeal nerve during thyroid surgery.

We describe how we found and managed this anatomical variant and we conduct a review of the most recent Literature about nonrecurrent laryngeal nerve.

\section{KEYWORDS}

Nonrecurrent Laryngeal Nerve, Thyroid Surgery, Emithyroidectomy.

Correspondins Author Information

Dr. Enrico Maria Amadei M.D.

Department of Otorhinolaryngology and Audiology, Infermi Hospital, Rimini, Italy, E-mail: enricomaria.amadei@libero.it.

Received: April 25, 2020; Accepted: May 20, 2020; Published: May 26, 2020

Copyright: (C) 2020 ASRJS. This is an openaccess article distributed under the terms of the Creative Commons Attribution 4.0 International license.

Citation: Enrico Maria Amadei, Christopher Fabbri, Marco Trebbi. Nonrecurrent Laryngeal Nerve: A Case Report and Literature Review. Med Clin Res Open Access, 2020;1(1):1-4.

\section{Introduction}

Typically, the inferior laryngeal nerves (ILN) are called recurrent (RLN). In fact, these nerves embrace the aortic arch on the left and the brachiocephalic trunk on the right, after having detached from the vagus nerve. Then they ascend along the dihedral angle of the tracheo-esophageal groove, to enter the larynx at the entry point, ie the cricothyroid junction.

However, we can have a nonrecurrent laryngeal nerve (NRLN) with an incidence rate of $0.3-0.8 \%$ on the right side and $0.004-0.04 \%$ on the left side (1). This occurrence is very rare and significantly increases the risk of nerve injury due to its non-recognition by the endocrine surgeon. In our experience of about 500 thyroid surgeries performed by the same senior surgeon during the period 2004-2019, this was our first time we found a right NRLN.

\section{Case Report}

We present the case of a 58-year-old man who was submitted to a right hemithyroidectomy for a unilateral thyroid goiter.

We performed a horizontal cervical incision of about 5 centimeters in length, 2 centimeters (about 2 fingerbreadths) above the jugulum (sternal notch). We followed a skin wrinkle. Then we raised the upper and lower skin flaps. We found the median alba line and we separated the subioid muscles, preserving them. So, we exposed the tracheal axis and the right cricothyroid muscle, as landmark for the the entry-point of the ILN. We then unglued the voluminous right lobe of the thyroid and we went to look for the ILN. The latter was not found in the tracheo-esophageal groove, with an ascending vertical trend, as usual. We found it instead with a very high horizontal course, starting directly from the vagus nerve on the right. We preserved it in its entirety, recognizing its functionality 
through a dedicated electric neurostimulator. The nerve ran in a single trunk and it was parallel to the inferior thyroid artery (Figures 1 and 2). We recognized it from its suspicious appearance and we verified its nature with the nerve stimulator.

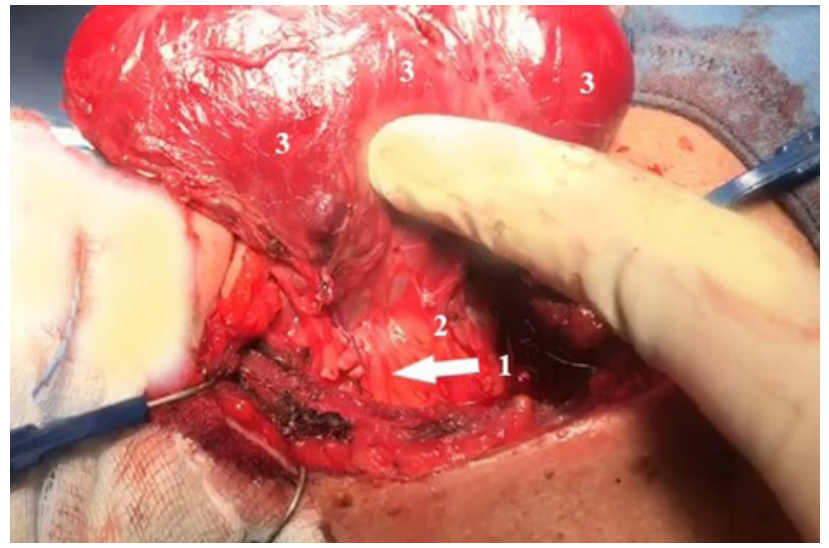

Figure 1: The right nonrecurrent laryngeal nerve (NRLN) runs in a single trunk and it is parallel to the inferior thyroid artery (1. Right NRLN; 2. Trachea; 3. Right thyroid lobe).

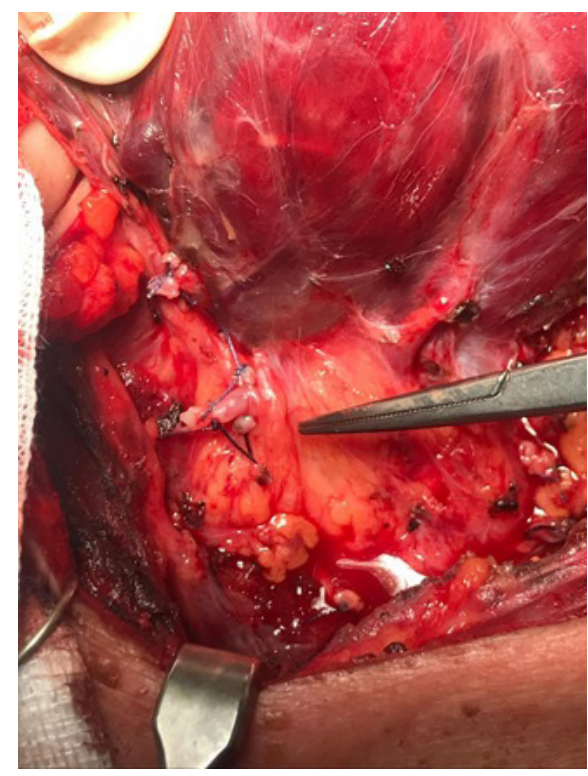

Figure 2: The right nonrecurrent laryngeal nerve (NRLN) runs in a single trunk and it is parallel to the inferior thyroid artery (see the tip of the tool).

We obviously were prepared for the occurrence of a NRLN, as the patient performed a CT scan of the neck before surgery. CT revealed a right arteria lusoria (lusory artery) (Figures 3 and 4). However, we had never previously found a NRLN during other surgical procedures. This anatomical variant evoked surprise and concern, as this time we did not know for sure the course of the ILN and we did not know if it presented as a single-trunk or with a multiple-branched course. In fact NRLN is a rare presentation, but the risk of intraoperative damage can reach $12.9 \%$ (2).

We then performed a right loboistmectomy and we finished the surgery. At the final histological examination, we found a right thyroid follicular adenoma.

Vol 1 Iss 1
The right NRLN was recognized and preserved. Its functionality was confirmed by end-of-surgery nerve stimulation and laryngoscopy postoperatively.

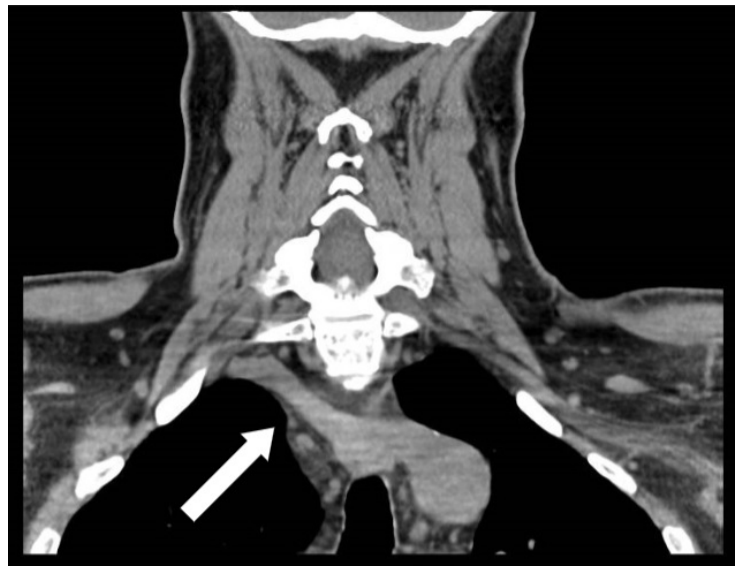

Figure 3: Pre-surgery CT scan highlights an aberrant right subclavian artery (see arrow).

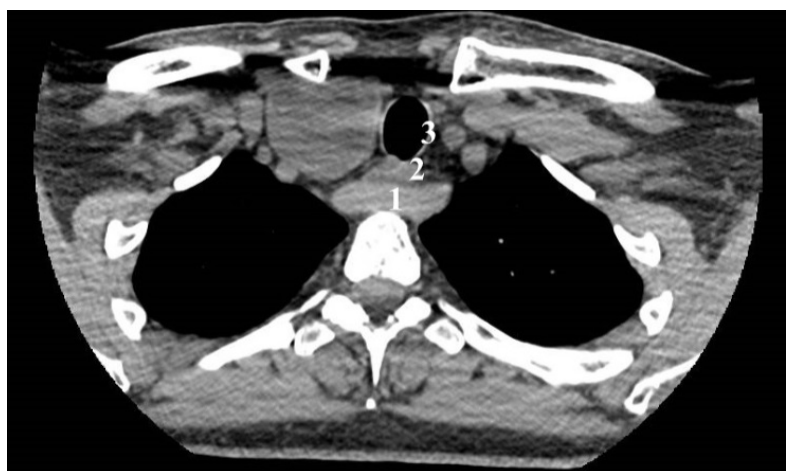

Figure 4: Pre-surgery CT scan: arteria lusoria has a retroesophageal course (1. arteria lusoria, 2. esophagus, 3. trachea).

\section{Discussion}

We performed a right hemithyroidectomy, and not a total thyroidectomy, on the advice of the Endocrinologist. We decided this for various reasons. Because the patient was not elderly; because the left thyroid lobe was healthy; because the cytological examination by fine needle aspiration citology (FNAC) was compatible with an indeterminate lesion with a low risk of malignancy (Tir-3A, according to Italian consensus for the classification and reporting of thyroid cytology 2014).

We know that in the case of thyroidectomy the main complications are of three types: 1 . hemorrhage, which is potentially fatal, since it is in the cervical region (3). 2. temporary/ permanent loss of one or more parathyroid glands, resulting in hypocalcemia, with risk of tetanus crises and arrhythmias. 3. finally the lesion of one or both ILN, with dysphonia (unilateral lesion) or dyspnea (bilateral lesion). The incidence rate of its monolateral lesion goes from 3 to $7 \%$ in Literature (4). Obviously the risks are halved in case of hemithyroidectomy.

The search for ILNs by the endocrine surgeon must be systematically conducted. The best way to save the ILNs is to look for them 
systematically, to identify them with certainty and to follow their course up to the entry-point.

The entry point is where the ILNs penetrate into the larynx, below the crico-thyroid muscle of each side. This is the only certain passage point for ILNs, but it is also the most risky point to search for ILNs. Therefore, surgeons usually search for ILNs at the tracheo-esophageal groove. From anatomical studies we know that more often on the left the ILN apperars as a single trunk, it is deep and it crosses more deeply the inferior thyroid artery. Otherwise on the right side, the ILN more easily presents an early branching, it is generally more superficial and generally it crosses the inferior thyroid artery passing through a deeper plane.

This case is very interesting for an endocrine surgeon, as it presents a rare anatomical variant related to the course of an ILN. We can typically find a NRLN on the right when an aberrant right subclavian artery is present. This artery is called "arteria lusoria". It detaches from the descending aorta and, moving towards the right, presents a more often retroesophageal (80\%), or pretracheal (5\%) or between trachea and esophagus (15\%) course (1).

During embryogenesis the nerves develop first and then the blood vessels. Therefore with the progressive cranio-caudal lengthening of the embryo, the ILNs are stretched downwards from the aortic arch on the left and the brachiocephalic trunk on the right. Then ILNs rise up to the entry-point, where they will go to innervate all the laryngeal muscles, with the exception of the cricothyroid muscles (innervated by the upper laryngeal nerves).

The right ILN does not remain trapped around its course, when an arteria lusoria is present. So, it is not dragged downwards, and therefore will not present a recurring pattern. So, it will proceed directly from the vagus nerve to the entry point.

Hong et al described a classification of NRLN with four traveling pattern types: (1) descending-the nerve descends from the vagus nerve trunk; (2) vertical-the nerve runs vertically to the cricothyroid joint; (3) ascending-the nerve runs upward to the cricothyroid joint; and (4) V-shaped-the nerve takes a downward course and runs upward to the cricothyroid joint (5). These Authors wrote about vertical trend, considering the floor-ceiling direction. Instead we are more used to considering the cranio-caudal direction as vertical. However according to their classification, in this case we found a NRLN with a vertical pattern.

The association between lusory artery and NRLN, however, is not always true. Cases of lusory artery associated with a RLN were described (6). In fact, prevalence of aberrant right subclavian artery is estimated to be around $0.5 \%$ to $2 \%$ of the population (7), while a right NRLN has a prevalence of $0.3-0.8 \%$ (1).

Therefore, the presence of an aberrant right subclavian artery must alert the surgeon, as it is probable the finding of a NRLN. It will therefore require even more attention than usual, to search for, identify and preserve the ILN. However, it is still possible to find Vol $\overline{1}$ Iss 1 an ILN in its classical site, even in presence of a lusory artery.

The possibility of a left NRLN is also reported in Literature. This occurrence can typically be found in cases of "situs inversus" $(1,2,8)$. The pre-operative CT study and the use of the nerve stimulator are two very useful tools for preserving the anatomical and functional integrity of the ILNs.

We always subject the patient to a CT scan before the thyroid surgery. This exam allows us to evaluate the localization of thyroid nodules, the size of the thyroid lobes and their relationship with blood vessels and the possible presence, as in this case, of the lusory artery. In other Centers the exclusive routine use of pre-operative ultrasound is described. It can well provide a pre-operative diagnosis regarding the presence of the lusory artery (9).

We use an intraoperative nerve monitoring, when performing a thyroidectomy. We routinely use a specific orotracheal tube cuff, positioning electrodes near vocal folds. We also use pulsed nerve stimulation, when it needed, in order to confirm the visual retrieval of ILNs. In Literature some Authors suspect the presence of a NRLN based on the stimulation of the vagus nerve. They suspect it when the distal stimulation of the vagus nerve does not evoke a motor response of the vocal cord, while they get the response with proximal stimulation. This can occur when there is a high ILN detachment, as happens with the NRLN $(10,11)$. However we are not use to stimulating the vagus nerve to search for ILN. Therefore we did not exploit this technique during our described hemithyroidectomy.

\section{Conclusion}

The ILNs are classically called recurrent nerves, but the endocrine surgeon must always keep in mind the possibility of their nonrecurrence.

The non-recurrence is very rare on the right side, and almost exceptional on the left one, but not impossible. We know from Literature that the presence of the lusory artery is almost always associated with the right NRLN, but not always.

\section{References}

1. Le VQ, Ngo QD, Ngo XQ. Nonrecurrent laryngeal nerve in thyroid surgery: Frequency, anatomical variations according to a new classification and surgery consideration. Head Neck, 2019;41(9):2969-2975.

2. Toniato A, Mazzarotto R, Piotto A, Bernante P, Pagetta C, et al. Identification of the nonrecurrent laryngeal nerve during thyroid surgery: 20-year experience. World J Surg, 2004;28:659-661.

3. Amadei E.M., Benedettini L, Piccin O. Two cases of cervical hemorrhage with upper airway obstruction: A life-threatening condition. Case Reports in Medicine, 2014.

4. Hayward NJ, Grodski S, Yeung M, Johnson WR, Serpell J. Recurrent laryngeal nerve injury in thyroid surgery: a review. ANZ J Surg, 2013;83:15-21. 
5. Hong KH, Park HT, Yang YS. Characteristic travelling patterns of nonrecurrent laryngeal nerves. J Laryngol Otol, 2014;128(6):534-539.

6. Willson TJ, Stevens J, Salinas N, Brennan JA. Aberrant position of nonrecurrent inferior laryngeal nerve: Report of 3 cases and a review of the literature. Ear Nose Throat J, 2016;95(6):E2631.

7. Henry BM, Sanna S, Graves MJ, et al. The Non-Recurrent Laryngeal Nerve: a meta-analysis and clinical considerations. PeerJ, 2017;5:e3012.

8. Masuoka H, Miyauchi A, Higashiyama T, Yabuta T, Kihara $\mathrm{M}$, et al. Right-sided aortic arch and aberrant left subclavian artery with or without a left nonrecurrent inferior laryngeal nerve. Head Neck, 2016;38(10):E2508-E2511.

9. Iacobone M, Citton M, Pagura G, Viel G, Nitti D. Increased and safer detection of nonrecurrent inferior laryngeal nerve after preoperative ultrasonography. Laryngoscope, 2015;125(7):1743-1747.

10. Tie Wang, Gianlorenzo Dionigi, DaqiZhang, XuehaiBian, Le Zhou, et al. Diagnosis, anatomy, and electromyography profiles of 73 nonrecurrent laryngeal nerves. Head Neck, 2018;40(12):2657-2663.

11. Gurleyik E, Gurleyik G. Nonrecurrent Laryngeal Nerve in the Era of Intraoperative Nerve Monitoring. Adv Med, 2016;2016:1606029. 